\title{
The formation and evolution of galaxies: what we may learn with the SKA
}

\section{Santiago García-Burillo*}

Observatorio Astronómico Nacional-OAN-Spain

E-mail: s.gburillo@oan.es

Exploring the formation and evolution of galaxies is one of the key science topics of the Square Kilometer Array (SKA). The unprecedented sensitivity of the SKA will enable us for the first time to map the cosmic distribution of neutral gas $\left(\mathrm{HI}, \mathrm{H}_{2}\right)$ in galaxies from the local universe up to cosmological distances. This unique observational legacy will help understand how galaxies from in dark matter (DM) overdensities, accrete gas in a quiescent mode or, alternatively, by interactions and mergers. In addition, by mapping the cosmic distribution of radiocontinuum emission, the SKA will provide the physical framework explaining how gas gets converted into stars in galaxies. Finally, the mapping of the cosmic distribution of baryonic oscillations by the SKA will shed light on the equation of state describing the dominant, but enigmatic, dark energy component in the universe.

2nd MCCT-SKADS Training School. Radio Astronomy: fundamentals and the new instruments August 26- September 4, 2008

Sigüenza, Spain

\footnotetext{
${ }^{*}$ Speaker.
} 


\section{The universe: general framework for galaxy formation}

In the last decades there has been considerable progress in understanding how galaxies may form, thanks to the parallel revolution in cosmological models. In cold dark matter (CDM) models, galaxies are expected to form hierarchically through the successive merging of small CDM spheroids. In this context, the limited knowledge on the behaviour of baryons during mergers is a major handycap. The physics of baryon assembling is exceedingly complex. The behaviour of the baryons in numerical simulations is simplisticly modelled using semi-analytical recipes. To falsify/verify these assumptions, observational constraints are paramount. However, there is a dearth of observational constraints to the behaviour of baryons in the process of galaxy formation. At early epochs most of the baryons are expected to be in the gas phase. Therefore the observation of the neutral gas component (atomic and molecular) in galaxies from cosmological distances down to the local universe is a prerequisite to understanding the formation and evolution of galaxies.

There is a number of open questions to be answered by the mapping of the cosmic distribution of baryonic matter in a wide range of redshifts:

- How and how quickly gas settles into DM halos? How are disks formed?

- What is the importance of mergers versus more quiescent cold gas accretion?

- Where are the missing dwarf galaxies?. Where are the missing massive galaxies?

- What is the physics of feedback and what explains downsizing ?

- When and how did the gas get converted into stars?

In the following we summarize how the SKA is expected to tackle with the resolution of the several issues mentioned above.

\section{The cosmic distribution of neutral atomic hydrogen (HI)}

\subsection{HI science today}

Neutral atomic hydrogen (HI) is by far the most abundante element in the universe. As such, observations probing $\mathrm{HI}$ are the best choice to trace galaxy evolution in a wide range of environments (clusters, isolated regions, voids...). The $21 \mathrm{~cm} \mathrm{HI} \mathrm{emission} \mathrm{line} \mathrm{is} \mathrm{an} \mathrm{excellent} \mathrm{tracer} \mathrm{of}$ the distribution and kinematics of neutral gas in elliptical galaxies and spiral disks. In particular, HI maps can be viewed as a fossil record of galaxy interactions and provide constraints to cold accretion models.

In the context of HI galaxy surveys undertaken with today's telescopes, the case of elliptical galaxies (E) deserves special attention. It is unclear if hierarchical assembly schemes can explain all the observational properties of ellipticals. While the classical merger sequence may explain the different HI morphologies found in E ([1]), slow but long-lasting cold accretion processes may also equally account for the morphology of $\mathrm{E}$ galaxies that were historically considered as merger remnant paradigms. This is the case of polar ring galaxies ([2]). An extension of these surveys to a larger number of objects will be a key to testing the different scenarios that explain the formation and evolution of massive spheroidal galaxies. 
Furthermore, HI lines in absorption, detected in radio-loud objects have been recently used to reveal massive gas outflows in a significant number of radiogalaxies ([3]). Blueshifted absorption lines of up to $2000 \mathrm{~km} / \mathrm{s}$-width have been detected in a few radiogalaxies, betraying the presence of massive and energetic HI outflows. These observations can provide crucial constraints to the feedback influence of activity on the neutral ISM. However, the number of objects that can be studied is scarce due to the sensitivity limitations to detect continuum emission to the required level.

The detection of huge HI halos in isolated spirals, of which NGC 891 is a representative example ([4]), betrays the existence of primordial massive gas reservoirs in galaxies (see Fig. 1). This gas may be continuously falling onto the disks of galaxies and thus feed star formation activity on long time scales. The detection of massive HI halos in a significant number of galaxies may provide an answer to the problem of the missing baryons. In addition, the study of $\mathrm{HI}$ emission in spirals can be also key to probing star formation laws and angular momentum transfer mechanisms in galaxy disks ([5];[6]). HI observations in an increasing number of galaxies is an essential piece of information in this type of analysis that probe evolutionary tracks in galaxies, and hence, require an improved statistics.

The Arecibo Fast ALFA (ALFALFA) HI survey of galaxies is a good illustration of how deep and how far we can get today in a typical HI detection and mapping experiment ([7]). The ALFALFA survey covers an area of $\sim 7000$ square degrees and detect a number of $\sim 30.000$ galaxies with typical $\mathrm{M}_{\text {gas }} \sim 10^{6}-10^{11} \mathrm{M}_{\odot}$ up to a redshift $\mathrm{z} \sim 0.06$. The deepest $21 \mathrm{~cm}$ observations have been able to detect HI emission in a galaxy of cluster Abell 963 with the Westerbork radiotelescope at redshift $\mathrm{z} \sim 0.2$ (see Fig. 2, taken from [8]). The role of mergers and cold accretion processes in galaxy evolution awaits to be ellucidated by extending the detection and mapping of $\mathrm{HI}$ emission and absorption to cosmological distances.

\subsection{HI science with the SKA}

The SKA will be the ultimate HI telescope. One of the main drivers of the SKA experiment is the possibility of studying the neutral hydrogen content of galaxies to cosmological significant distances $(\mathrm{z} \sim 2)$. This goal can be reached thanks to the enormous gain in sensitivity: the SKA would have a collecting area $\sim$ two orders of magnitude larger than existent radiotelecopes. With this sensitivity goals at hand, the SKA would be able to detect Milky Way-like galaxies out to $\mathrm{z} \sim 1-$ 1.5 and large spiral galaxies like M 101 out to $\mathrm{z} \sim 2.5$, after a typical integration time of 12 hours (see [9]). Besides its high-sensitivity capabilities, the SKA will combine a large field-of-view ( $\sim 50$ square degrees at $<1 \mathrm{GHz})$ with a wide frequency coverage and bandwith $(0.1-25 \mathrm{GHz})$. Thanks to these unprecedented capabilities, the SKA will boost the statistics of the number of HI galaxies detected to $\sim 10^{7}$ targets per square degree out to $\mathrm{z} \sim 5$ in just $\sim 15$ days of regular observations. The improved statistics will allow us to observe changes in the HI mass function of galaxies as function of redshift, an information to be confronted with the predictions of theoretical models that predict a significant evolution to be tested.

By mapping the cosmic distribution of radiocontinuum (both thermal and non-thermal) emission to cosmological distances, the SKA will shed light on the physical framework explaining how gas gets converted into stars in galaxies. As an illustration, the SKA will be able to detect the radiocontinuum emission of a moderate star forming galaxy $\left(\sim 10 \mathrm{M}_{\odot} \mathrm{yr}^{-1}\right)$ out to a redshift $\mathrm{z} \sim 4$ 


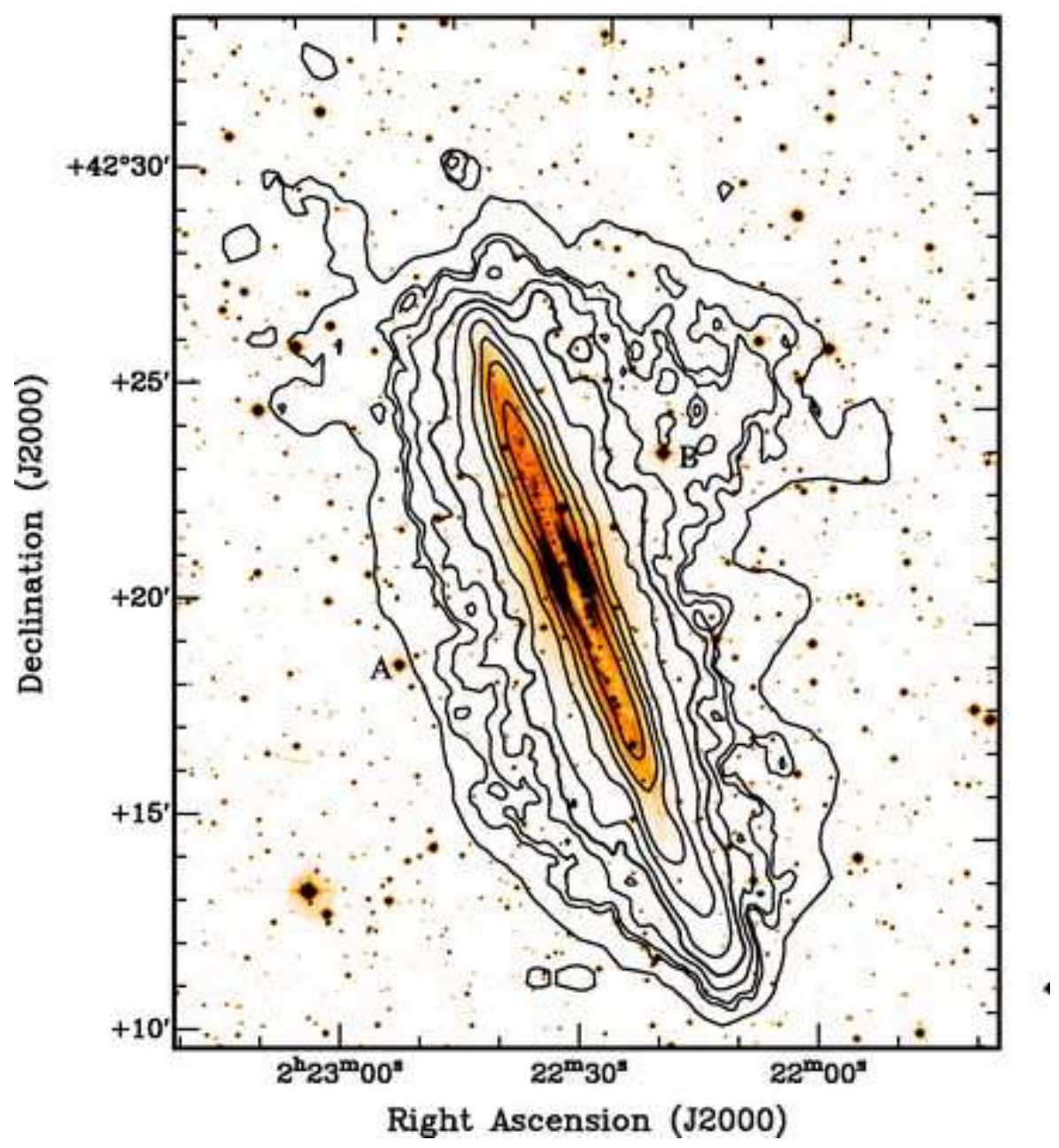

Figure 1: HI emission map of the nearby edge-on spiral galaxy NGC 891 (adapted from [4]) shows huge HI halo. Data obtained by the Westerbork radio telescope (WSRT). Level contours are 0.005, 0.01, 0.02, 0.05, $0.1,0.2,0.5,1.0,2.0$ and $5.0 \cdot 10^{21} \mathrm{~cm}^{-2}$.

after 8 hours of integration (see [9]). This will enable us to make a significant step forward in our understanding of star formation laws in galaxies.

\section{The cosmic distribution of molecular hydrogen $\left(\mathrm{H}_{2}\right)$}

Molecular gas is the fuel of star formation activity in galaxies. Current millimeter radiotelescopes have been able to map the emission of $\mathrm{CO}$ and that of other molecular species, and thus trace the content, distribution and kinematics of molecular gas in an ample number of nearby galaxies. The study of CO line emission in nearby spiral disks have been used to study star formation laws in galaxies ( [10]). Furthermore, high-spatial resolution CO observations, obtained with mm-interferometers, have been instrumental in the study of AGN feeding mechanisms in the local universe ([11]). The high-sensitivity of current mm-interferometers have also allowed to detect the emission of molecular gas in galaxies situated at redshifts beyond $\mathrm{z} \sim 1$. Among the most re- 

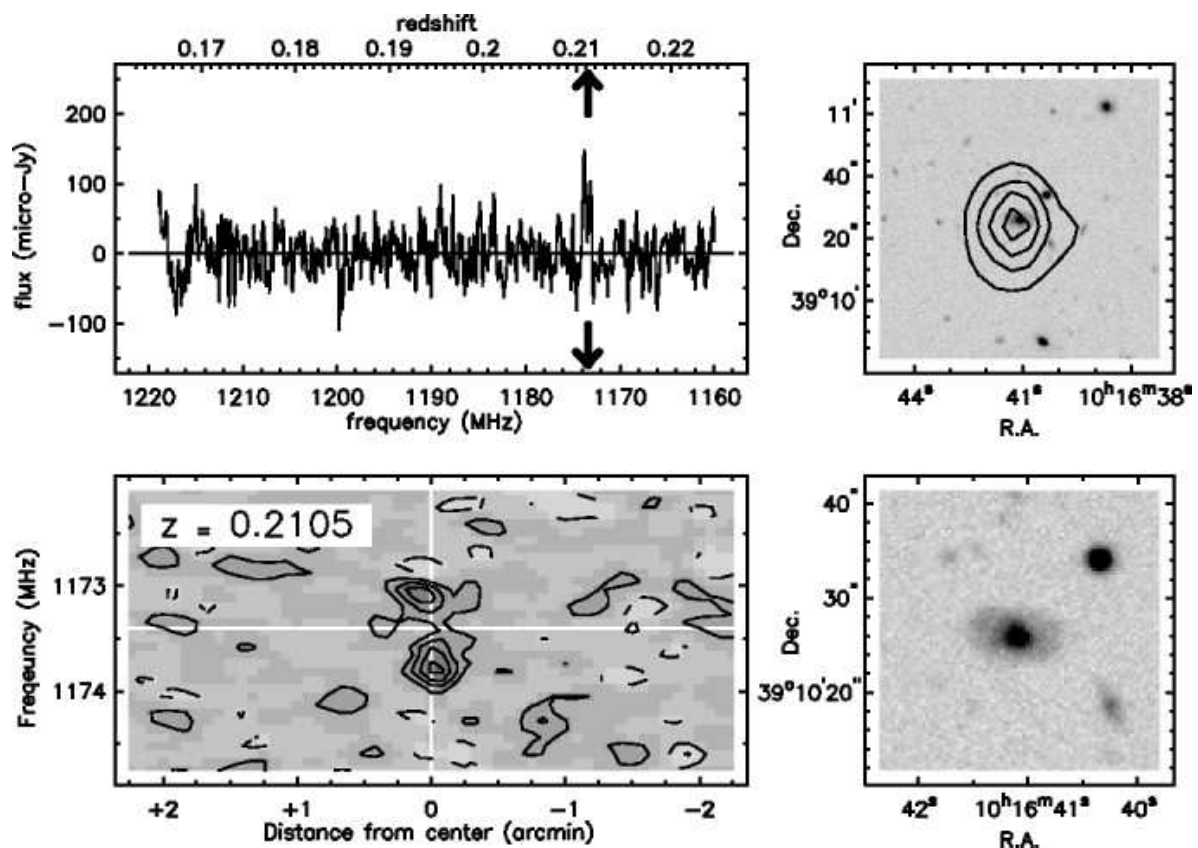

Figure 2: HI detection at redshift $\mathrm{z} \sim 0.2$, a spatially resolved galaxy in cluster Abell 963. Top left shows HI spectrum. Bottom left: Position-velocity HI diagram along major axis extracted from the H I data cube. Top right: Integrated HI contours superimposed on R-band image (gray scale). Bottom right: Enlargement of the optical image. Figure taken from [8].

markable detections, it is worth noting the case of SDSS J1148+5251, the highest redshift quasar currently known at $\mathrm{z} \sim 6.4$ ( [12]).

The detection of high-redshift $\mathrm{CO}$ emission has been limited to a reduced $(\sim 100)$ number of galaxies, however. Many of the so called high-redshift zoo members (e.g., Sub Millimeter Galaxies (SMGs), Lyman Break Galaxies(LBGs), Star Forming Galaxies (SFGs) and quasars (QSOs)) are gravitationally lensed systems, and thus their emission is naturally amplified. Current models of galaxy formation and evolution would benefit from the observation of the normal population of star forming galaxies at high redshift.

Besides being a powerful HI radiotelescope, the SKA will also be able to probe the distribution of molecular hydrogen in galaxies to cosmological distances. As an example, the cumulative number of $\mathrm{CO}(1-0)$-detected galaxies at $v \sim 22 \mathrm{GHz}$ would amount to $\sim 10^{3}$ per square degree after one hour of integration time ([13]). In this context, it is worth noting that the SKA telescope may be a nice complement to the capabilities of the Atacama Large Millimeter Array (ALMA). In particular, while ALMA will probe the high rotational lines of CO and other molecules in the high-redshift universe, the SKA will provide information on the lower rotational lines of the same species. The combination of SKA and ALMA observations is nedeed to get a handle on the spectral energy distribution of molecular lines in a large sample of galaxies and, also, for a wide range of redshifts. 


\section{The cosmic distribution of baryonic oscillations}

Baryonic Acoustic Oscillations (BAO) have a preferred spatial scale from the era of recombination. The comparison between BAOs at different $\mathrm{z}$, through galaxy redshift surveys to be done by the SKA, with CMB fluctuations gives an estimate of the equation of state of the dark energy component in the universe. The information gained from the study of the wiggles in the distribution of baryons as a function of redshift is orthogonal with respect to that obatined in the study of CMB fluctuations. The SKA is a specially designed instrument to measure the power spectrum of the baryonic oscillations in the universe at different redshift ranges ( [14]). A detailed review on this key science topic for the SKA has been written by [15].

\section{References}

[1] Oosterloo, T. A., Morganti, R., Sadler, E. M., van der Hulst, T., \& Serra, P. 2007, A\&A, 465, 787

[2] Macciò, A. V., Moore, B., \& Stadel, J. 2006, ApJL, 636, L25

[3] Morganti, R., Oosterloo, T. A., Emonts, B. H. C., van der Hulst, J. M., \& Tadhunter, C. N. 2003, ApJL, 593, L69

[4] Oosterloo, T., Fraternali, F., \& Sancisi, R. 2007, AJ, 134, 1019

[5] Haan, S., Schinnerer, E., Mundell, C. G., García-Burillo, S., \& Combes, F. 2008, AJ, 135, 232

[6] Walter, F., Brinks, E., de Blok, W. J. G., Bigiel, F., Kennicutt, R. C., Thornley, M. D., \& Leroy, A. 2008, AJ, 136, 2563

[7] Giovanelli, R., et al. 2005, AJ, 130, 2598

[8] Verheijen, M., van Gorkom, J. H., Szomoru, A., Dwarakanath, K. S., Poggianti, B. M., \& Schiminovich, D. 2007, ApJL, 668, L9

[9] van der Hulst, J. M., Sadler, E. M., Jackson, C. A., Hunt, L. K., Verheijen, M., \& van Gorkom, J. H. 2004, New Astronomy Review, 48, 1221

[10] Bigiel, F., Leroy, A., Walter, F., Brinks, E., de Blok, W. J. G., Madore, B., \& Thornley, M. D. 2008, $A J$, 136, 2846

[11] García-Burillo, S., Combes, F., Schinnerer, E., Boone, F., \& Hunt, L. K. 2005, A\&A, 441, 1011

[12] Walter, F., Carilli, C., Bertoldi, F., Menten, K., Cox, P., Lo, K. Y., Fan, X., \& Strauss, M. A. 2004, ApJL, 615, L17

[13] Blain, A. W., Carilli, C., \& Darling, J. 2004, New Astronomy Review, 48, 1247

[14] Glazebrook, K., et al. 2007, Cosmic Frontiers, 379, 72

[15] Rawlings, S., Abdalla, F. B., Bridle, S. L., Blake, C. A., Baugh, C. M., Greenhill, L. J., \& van der Hulst, J. M. 2004, New Astronomy Review, 48, 1013 